\title{
Collaborative Environmental In Situ Data Collection: Experiences and Opportunities for Ambient Data Integration
}

\author{
David Thau \\ Google, Inc., 1600 Amphitheatre Parkway, Mountain View, CA 94043 \\ thaulgoogle.com
}

Collaborative environmental in situ data collection occurs when a team of investigators goes into the field together to collect environmental data. These data might be necessary, e.g., for a biodiversity inventory, compilation of a soil density map, or to estimate above-ground forest carbon stocks. Investigators will often arrive at a location and disperse, collecting data, and then compiling it either in the field, or at a later time. Typically, an area will be divided into a set of plots, and within those, subplots. Teams of investigators will visit each of these plots with standardized forms and specialized equipment for collecting the data of interest. For example, in a forest inventory, investigators might collect data about the diameter and species of the trees in the forest, the trees' health, fire damage and soil quality at the plot, proximity to roads, and whether any logging has taken place.

Plots are often hard to reach, involving treks through dense forest, river crossings, and other obstacles. This constrains the types of materials that may be brought into the field. Once in the field, working conditions can be very uncomfortable, constraining the amount of time spent in the field. To minimize the time necessary to collect their data, teams often parallelize data collection. In some cases, each person visits a subplot and records all the data there, and the data are later combined. In other cases, one person is given the task of recording data while the others yell out measurements. Once the data have been collected, they must be validated. This validation includes verifying that numbers are within reasonable ranges, ensuring that recorded species are conceivably located in the given region, and checking the uniqueness of identifiers. Some of this validation may be done in the field, while others, such as checking system-wide identifier uniqueness, require access to database that may not easily be brought into the field.

While most people engaging in collaborative in situ data collection use paper forms to collect their data, the task lends itself to the application of more recently developed technologies, such as PDAs, smart phones, and wireless networks. This talk will provide details about collaborative environmental in situ data collection, and discuss general challenges involved in bringing non-paper-based technology into the field, as well as offer some suggestions for how these difficulties may be mitigated. Most importantly, the talk will present a number of places where ambient data integration may be applied to further reduce the amount of time involved in collecting in situ data. Finally, the talk will conclude by generalizing this work to areas outside of environmental data collection. 